\title{
Management of Delayed Union of Tibial Shaft Fractures by Platelet Rich Plasma
}

M.A.Mashhour, M.E.Al-Ashhab , E.M.Bayoumi and A.M.Samy

Orthopedic Surgery Dept., Faculty of Medicine, Benha Univ., Benha, Egypt

E-Mail:ahmedsamyhabib@gmail.com

\begin{abstract}
The aim was to evaluate the results of platelet rich plasma (PRP) infusion in postponed joined tibial cracks. Development factors have a part in angiogenesis and osteogenesis by upgrade of separation of undifferentiated mesenchymal cells. Platelets are wealthy in these development factors. The point of use of PRP in crack site to upgrade osteogenesis. Sixteen patients with postponed joined tibial shaft cracks were overseen by PRP infusion and surveyed from April 2018 till April 2019 at Banha University Hospital. Postoperative appraisal was done clinically and radiologically by radiological association size of tibia (RUST). Last evaluation was done at a half year postoperatively and the outcomes were as per the following: Complete crack recuperating was acquired in 14 patients $(87.5 \%)$ with mean time $3.07 \pm 0.92$ with range 2 - a half year, and just two patients (12.5\%) didn't show any association after development for a half year and was named as disappointment of treatment. No complexities identified with careful procedure were noticed. A few patients created subcutaneous expanding of not many centimeters in width at the infusion site. Platelet-rich plasma is a protected and powerful treatment of deferred association tibial shaft fractures.
\end{abstract}

Keywords: Delayed union, PRP.

\section{Introduction}

Fracture mending is a complex physiological cycle including a planned collaboration of haemato-poietic and insusceptible cells inside the bone marrow, related to vascular and skeletal cell forerunners. Various cytokines and development factors are powerful at various phases of this cycle [1].

In spite of this efficient regenerative reaction, recuperating issues are seen in roughly $10 \%$ of breaks [2].

A deferred association is non joined break that didn't show any radiological association (crossing over callus on 3 cortices) following 3 months and before a half year of legitimate obsession or that has not been available for enough time to fulfill standard time for association [3].

Typical recuperating is restricted because of a lacking natural reaction at the crack site. Additionally, deficient mechanical strength is an explanation which prompts deferred association [4].

A few organic danger factors have been related with deferred association and nonassociation as mature age, diabetes, smoking, healthful lacks, and the utilization of mitigating specialists [5].

In treatment of postponed association, organic and mechanical components should be assessed. In the wake of accomplishing mechanical strength with interior or outside obsession, an endeavor is made to accomplish association with enlistment of organic components [6].

Neighborhood organic improvement systems incorporate the utilization of osteogenic materials as autologous bone marrow and peptide flagging particles and furthermore the utilization of osteoinductive and morphogenetic factors as bone morphogenetic proteins [5].

Platelet Rich Plasma (PRP) Injection is one of the new organic upgrade patterns in the administration of postponed association and non-association cracks. PRP acts to invigorate normal mending ventures through development factors contained in the platelets. PRP applied to the break site quickens the physiological recuperating measure, offers help for the association of cells, diminishes torment and has an enemy of bacterial impact [6].

Platelet-rich plasma (PRP), which is a characteristic concentrate of autologous development factors, is currently being generally tried in various fields of medication for its prospects in supporting the recovery of tissue with low recuperating potential. The bone regenerative impact of PRP is tweaked by development factors, for example, plateletinferred development factor, insulin-like development factor, and changing development factor (TGF)- B. These components help in setting off chondroblastic and osteoblastic separation just as the creation of new bone lattice [7].

Thus, autologous PRP, comprising of a concentrated suspension of platelets in a restricted measure of plasma, has picked up an expanding notoriety as a fruitful crack mending treatment [8]. The simple arrangement conventions, biosafety and flexibility of platelet-rich arrangements and their diminished expense have empowered 
their helpful use for incitement of tissue mending and bone regeneration [9].

\section{Patients and methods}

This is an interventional study that was done on 16 patients after acknowledgment of clinical moral panel in workforce of medication in Banha college. Every one of these patients have deferred association of tibial crack, and every one of them were educated regarding the strategy. The investigation was during the period from April 2018 to April 2019 in division of muscular medical procedure in Banha college clinic.

\subsection{Inclusion standards}

Patients with clinical and radiological indications of postponed association of tibial shaft breaks.

Stable inner obsession or stable decrease with mortar projecting.

Acceptable arrangement of crack parts

Postponed association was

characterized as a break of span over 3 months after injury or time from last crack site activity, which had not indicated reformist proof of recuperating.

\subsection{Exclusion measures}

Open breaks and cracks related with neurovascular injury.

Infected cracks.

Pathological cracks.

Intra-articular breaks or cracks with insecure obsession.

The included patients were $13(81.25 \%)$ guys and $3(19.75 \%)$ females with mean age $36.9 \pm 12.56$ years going from 20 to 56 years.

The patients were introduced after the medical procedure with postponed association after $3.67 \pm 0.72$ months of crack obsession with range from 3 to 5 months which didn't show critical impact on association. Thirteen $(81.25 \%)$ patients were fixed by intramedullary nail, two $(12.5 \%)$ were fixed by plate and screws and one (6.25) was fixed by Ilizarov. Eleven $(68.75 \%)$ patients went through shut decrease of the break and five $(31.25 \%)$ were went through open decrease. Six $(37.5 \%)$ patients indicated deferred demonstrated postponed association with atrophic break edges. Which shows that impact of infusion was preferred on atrophic non-association over hypertrophic non-association. As indicated by comorbidities, four (25\%) patients had diabetes mellitus and twelve $(75 \%)$ patients had no co-morbidities.

\subsection{Platelets Rich Plasma (PRP) arrangement}

PRP is set up by a cycle known as differential centrifugation. In differential centrifugation, increasing speed power is acclimated to residue certain cell constituents dependent on various explicit gravity. There are numerous methods of getting ready PRP, it very well may be set up by the PRP strategy or by the Buffy-coat technique. We utilized PRP technique, first we draw around (50 to $75 \mathrm{ml}$ ) by venipuncture and afterward we gather around $15 \mathrm{ml}$ conelike cylinders containing anticoagulant which was sodium citrate. An underlying centrifugation to isolate red platelets (RBC) (axis at $160 \mathrm{~g}$ for $15 \mathrm{~min}$ at 20c) this is trailed by a subsequent centrifugation to think platelets (rotator at $400 \mathrm{~g}$ for $15 \mathrm{~min}$ at $20 \mathrm{c}$ ), which are suspended in the littlest last plasma volume. Entire blood is at first gathered in cylinders that contain anticoagulants. The primary turn step is performed at steady speeding up to isolate RBCs from the excess entire blood volume. After the principal turn step, the entire blood isolates into three layers: an upper layer that contains generally platelets and WBC, a middle of the road flimsy layer that is known as the buffy coat and that is turn should be only sufficient to help in development of delicate pellets (erythrocyte-platelet) at the lower part of the cylinder. The upper part of the volume that is made generally out of PPP (platelethelpless plasma) is eliminated. Pellets are homogenized in lower $1 /$ third $(5 \mathrm{ml}$ of plasma) to make the PRP [10].

\subsection{Injection technique}

Injections are most often performed under imaging guidance under complete aseptic conditions. Injection will be directly in the fracture site and also around it. 


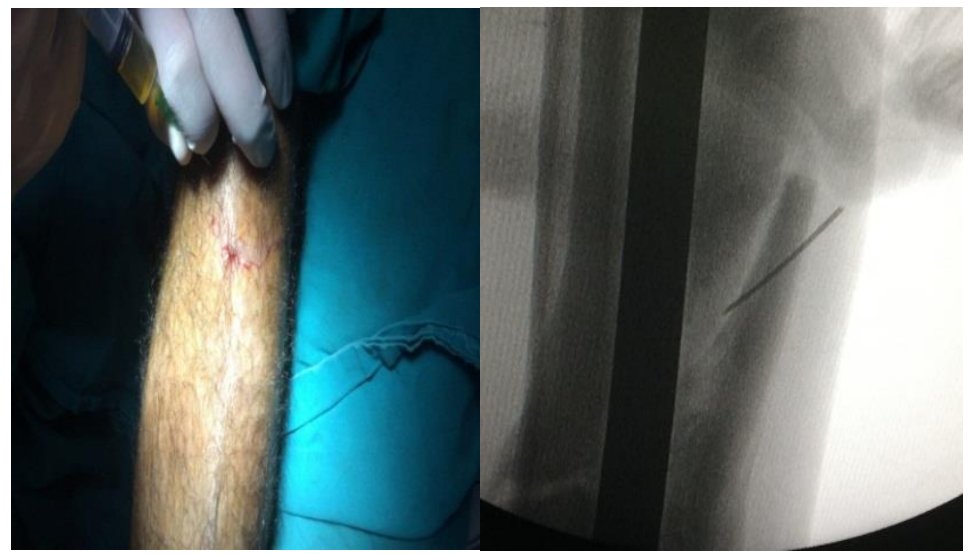

\section{Post injection Protocol}

Patients were instructed to reduce movements of their knee and ankle joints for the next 24 hours and to use paracetamol as analgesic. It is advised not to use NSAIDs as they may impair platelet function [11].

\section{Post-injection evaluation}

All the patients were assessed clinicoradiologically every week for one month to exclude infection then monthly for follow up of healing of fracture till 6 months. The absence of localized tenderness, abnormal mobility and pain were considered clinical criteria for union. Radiological union was assessed with the help of anteroposterior,
PRP injection.

lateral and two oblique $\mathrm{x}$-rays at each visit. Radiographic union was evaluated by radiographic union scale in tibial fracture (RUST) score. Each tibial cortex (anterior, posterior, medial and lateral) was assigned a RUST score of 1 to 3 , based on the appearance. A cortex with a visible fracture line and no callus was given a score of 1 , a cortex where callus and a visible fracture line was present was scored as 2 , and a cortex with bridging callus and no fracture line within the callus bridge was scored as 3. The scores of all cortices were then combined to give a minimum score of 4 (definitely not healed) and a maximum of 12 (completely healed) [12].

Table (1) Radiographic union scale in tibial fracture (RUST) score [12].

\begin{tabular}{lll}
\hline Score per cortex & Callus & Fracture line \\
\hline 1 & ABSENT & Visible \\
2 & PRESENT & Visible \\
3 & PRESENT & Invisible \\
\hline
\end{tabular}

\section{Results}

Complete fracture healing was obtained in 14 patients $(87.5 \%)$ with mean time $3.07 \pm 0.92$ with range 2 - 6

months, and only two patients (12.5\%) did not show any union after follow up for six months and was labeled as failure of treatment.

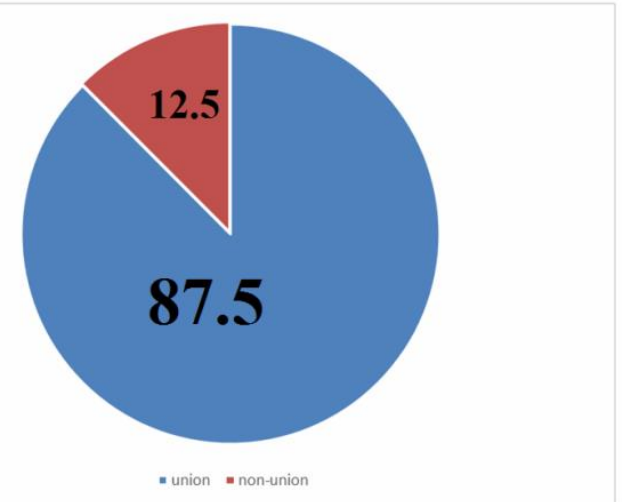

Pie-chart showing Assessment of post-operative union.

Radiographic union was assessed pre- and post-injection by RUST score. The pre-injection RUST ranged from 4:6 with mean 4.81. One month after injection its range became 4:9 with mean 6.06. Three months later, the range was 4:11 with mean 9.06. After six months, the range was $4: 12$ with mean 10.62 . 


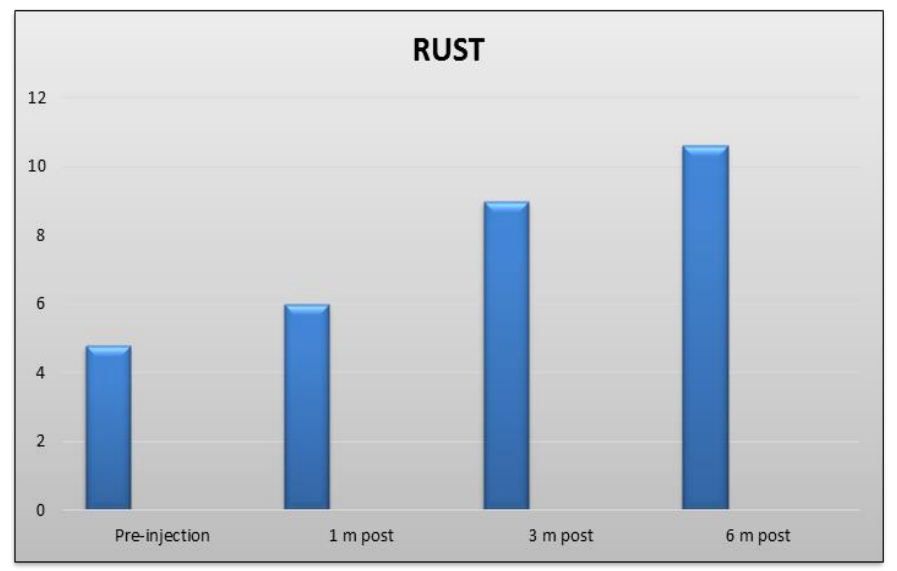

Showing the RUST of patients pre and post injection.

\section{Discussion}

Nonunion and postponed association of the broke bones is one of the regular issues experienced in muscular health practice. The rate of non-association is more in patients of cutting edge age, those with fringe vascular illnesses, patients on steroids or immunosuppressants and in people with nearby twisted disease. While it is critical to recognize and treat the reason for non-association and deferred association, for example, bone uniting in instances of bone misfortune, antimicrobials in instances of contamination and appropriate interior obsession in instances of precarious obsession, there are sure organic specialists which may rush the cycle of association in broke bones. These natural specialists incorporate goal concentrate of bone marrow, platelet rich plasma, fibroblast development factors, undeveloped cells and bone morphogenic protein. To the extent utilization of these organic specialists in the patients of deferred and nonunion is worried there is huge expansion in light of a legitimate concern for scientists.

The working meaning of platelet-rich plasma (PRP) is a volume of autologous plasma that has a platelet grouping of $1,000,000$ platelets in a $5-\mathrm{ml}$ volume of plasma. Exogenous use of platelet-related development factors has been appeared to animate multiplication of osteoblasts in culture and has been exhibited to invigorate the enlistment and expansion of osteoblasts.

In view of function of development factors on angiogenesis and osteogenesis by upgrade of separation of undifferentiated mesenchymal cells, our investigation was done on 16 patients and $87.5 \%$ was accomplished as an association pace of postponed association tibial crack.
Mean season of association after infusion was $3.07 \pm 0.92$ with range 2-6 months .

Malhotra et al did his examination on 35 patients, and he accomplished $88.57 \%$ as an association rate in interim 2.4 with range 3-4 months [13].

Bielecki et al did his examination on 12 patients, and he accomplished $100 \%$ as an association rate in interim 2.3 with range 23 months [14].

Dallari et al has discovered that the utilization of PRP improve the callus arrangement of high tibial osteotomy and reduction the recuperating time frame [15].

Then again, Joost et al has discovered that there was no any critical distinction subsequent to utilizing the PRP in bone prattle recuperating [16].

In our examination and other related investigations, there were no entanglements identified with percutaneous infusion method, aside from subcutaneous expanding of not many centimeters in width at the infusion site which showed up in couple of patients during infusion and settled in no time, which uncovers the security of that technique.

\subsection{Study limitations}

This study has many limitations and some points of weakness. Firstly, the study assessed the outcomes of PRP injection without the use of control group. Secondly, we are not able to conclude whether the method will be effective in the presence of bone defects as we selected only fractures with near total contact between the fracture edges. Thirdly, we used centrifugation for concentrating platelets, which may lead to the fragmentation of the platelets. Fourthly, small number of cases and short period of follow up which may not give accurate results. 


\subsection{Future prospective and recommendations}

To achieve acceptable clinical results, the current study recommends:

Proper patient selection. PRP injection in the treatment of delayed union tibial fractures in absence of any mechanical cause or infection.

It is better to use ultrafiltration to prepare PRP which may be a more efficient method than centrifugation.

\section{Conclusion}

In conclusion PRP injection is useful in the treatment of delayed union tibial fractures. It is easy to perform and effective treatment.

\section{References}

[1] E.Solheim, Growth factors in bone. International Orthopaedics, Vol.22, PP.410-416,1998.

[2] T.A.Einhorn,Enhancement of fracturehealing. Journal of Bone and Joint Surgery. Am, Vol.77-A, PP.940956,1995.

[3] L.S.Phieffer and J.A.Goulet,Delayed unions of the tibia. Journal of Bone and Joint Surgery, Vol. 88-A, PP.206216,2006.

[4] M.K.Sen and T.Miclau,Autologous iliac crest bone graft: should it still be the gold standard for treating nonunions? Injury, Vol.38, PP.75-80,2007.

[5] T.Einhorn and L.C.Gerstenfeld,Fracture healing mechanisms and interventions. Nature Reviews Rheumatology, Vol.11, PP.45-54,2015.

[6] M.Sánchez, E.Anitua, G. Orive, I. Mujika, Platelet-rich therapies in the treatment of orthopaedic sport injuries. Sports Medicine, Vol. 39, PP.345354,2009.

[7] S.W.Xu, R.Yu and G.F.Zhao, Early period of fracture healing in ovariectomized rats. Chinese Journal of Traumatology, Vol.6, PP.160-166,2003.

[8] R.Simman, A.Hoffmann and J.R.Bohinc, Role of platelet-rich plasma in acceleration of bone fracture healing. Annals of Plastic Surgery, Vol.61, PP.337-344,2008.

[9] Roop Singh, Rajesh Rohilla, Jeetesh Gawande and K.S.Paramjit, role of platelet-rich plasma in healing of acute diaphyseal fractures of the femur. Chinese Journal of Traumatology, PP.39-40,2017.

[10] A.S.Wasterlain, H.J.Braun and J.L.Dragoo,Contents and Formulations of Platelet Rich Plasma. Platelet Rich
Plasma in musculoskeletal practice, Vol.1, PP. 7,2020.

[11] A.Moshiri and A.Oryan, Role of platelet-rich plasma in soft and hard connective tissue healing: an evidencebased review from basic to clinical application. Hard Tissue, 2013.

[12] J.M.Leow, N.D.Clement, T.Tawonsawatruk, C.J.Simpson ,The radiographic union scale in tibial(RUST) fractures. Bone and joint research, Vol.5, PP. 116-121,2016.

[13] R.Malhotra, V.Kumar, B.Garg, R.Singh, Role of autologous plateletrich plasma in treatment of long-bone nonunions. Journal of Musculoskeletal Surgery.; 99(3), PP.243-8,2015.

[14] T.Bielecki, T.S.Gazdzik and T. Szczepanski, Benefit of percutaneous injection of autologous plateletleukocyte-rich gel in patients with delayed union and nonunion. European Surgical Research, Vol.40(3), PP.28996,2008.

[15]D.Dallari, L.Savarino, C.Stagni, E.Cenn i, Enhanced tibial osteotomy healing with use of Bone Grafts Supplemented with Platelet Gel or Platelet Gel and Bone Marrow Stromal Cells. American Journal of Bone and Joint Surgery, Vol.89(11), PP.2413-20,2007.

[16] C.Joost, W.Joost, A.H.Anita , V.a.n.Mark Appeldorn, D.J.Bruijn, No positive bone healing after using platelet rich plasma in a skeletal defect. International Orthopaedics ., Vol. 36(10), PP.2113-2119, 2012. 